\title{
THOMASSEN'S CHOOSABILITY ARGUMENT REVISITED
}

\author{
DAVID R. WOOD AND SVANTE LINUSSON
}

\begin{abstract}
Thomassen (1994) proved that every planar graph is 5-choosable. This result was generalised by Škrekovski (1998) and He et al. (2008), who proved that every $K_{5}$-minor-free graph is 5 -choosable. Both proofs rely on the characterisation of $K_{5}$-minor-free graphs due to Wagner (1937). This paper proves the same result without using Wagner's structure theorem or even planar embeddings. Given that there is no structure theorem for graphs with no $K_{6}$-minor, we argue that this proof suggests a possible approach for attacking the Hadwiger Conjecture.
\end{abstract}

\section{INTRODUCTION}

In 1943, Hadwiger [2] made the following conjecture, which is widely considered to be one of the most important open problems in graph theory]; see [14] for a survey.

Hadwiger Conjecture. Every $K_{t}$-minor-free graph is $(t-1)$-colourable.

The Hadwiger Conjecture is true for $t \leq 6$ [9, 10] and unsolved for $t \geq 7$. In general, $c t \sqrt{\log t}$ is the best known upper bound on the chromatic number of $K_{t}$-minor-free graphs, for some constant $c[7,12]$. This result is proved as follows. A graph $G$ is d-degenerate if every subgraph of $G$ has a vertex of degree at most $d$. Every $d$-degenerate graph is $(d+1)$ colourable - choose a vertex $v$ of degree at most $d$, apply induction to $G-v$, and colour $v$ with one of the colours not present in its neighbourhood. Kostochka [7] and Thomason [12] independently proved that every $K_{t}$-minor-free graph is $c t \sqrt{\log t}$-degenerate, and is thus $c t \sqrt{\log t}$-colourable. The following conjecture remains unsolved.

Weak Hadwiger Conjecture. There is a constant $c$ such that every $K_{t}$-minor-free graph is ct-colourable.

There are (at least) two major obstacles to overcome in a proof of the Hadwiger Conjecture or the Weak Hadwiger Conjecture:

- There are $K_{t}$-minor-free graphs with minimum degree $c t \sqrt{\log t}$ for some constant c. Therefore the above degeneracy-based algorithm fails.

Date: May 21, 2018.

2000 Mathematics Subject Classification. 05C83 graph minors, 05C15 graph coloring.

David Wood is a QEII Research Fellow supported by the Australian Research Council.

Svante Linusson is a Royal Swedish Academy of Sciences Research Fellow supported by a grant from the Knut and Alice Wallenberg Foundation.

${ }^{1}$ All graphs in this paper are undirected, simple and finite. We employ standard graph-theoretic terminology and notation [1]. 
- For $t \geq 6$, there is no known precise structural characterisation of $K_{t}$-minor-free graphs (and even for $t=6$ the situation seems hopeless).

This paper suggests a possible approach around these two obstacles.

A list-assignment of a graph $G$ is a function $L$ that assigns to each vertex $v$ of $G$ a set $L(v)$ of colours. $G$ is $L$-colourable if there is a colouring of $G$ such that the colour assigned to each vertex $v$ is in $L(v) . G$ is $k$-choosable if $G$ is $L$-colourable for every list-assignment $L$ with $|L(v)| \geq k$ for each vertex $v$ of $G$. If $G$ is $k$-choosable then $G$ is also $k$-colourable - just use the same set of $k$ colours for each vertex. Also note that every $d$-degenerate graph is $(d+1)$-choosable. See [19] for a survey on list colourings. Kawarabayashi and Mohar [6] made the following conjecture:

Weak List Hadwiger Conjecture. There is a constant $c$ such that every $K_{t}$-minor-free graph is $c t$-choosable.

Kawarabayashi and Mohar [6] wrote that they believe the Weak List Hadwiger Conjecture holds for $c=\frac{3}{2}$. Wood [18] conjectured it with $c=1$.

List Hadwiger Conjecture. Every $K_{t}$-minor-free graph is $t$-choosable.

For $t \in\{2,3,4\}$, every $K_{t}$-minor-free graph is $(t-2)$-degenerate, and thus is $(t-1)$ choosable. Now consider the $t=5$ case. Thomassen [13] proved that every planar graph is 5-choosable, and Voigt [15] constructed planar graphs that are not 4-choosable. Thomassen's result was generalised by Škrekovski [11] and He et al. [3] as follows:

Theorem 1 ([3, 11] ). Every $K_{5}$-minor-free graph is 5-choosable.

One feature of Thomassen's proof is that it does not depend on the degeneracy of planar graphs. Thus list colourings provide a potential route around the first obstacle above. See [6, 18] for more concrete examples of this idea. The second obstacle remains. In particular, Thomassen's proof relies heavily on the structure of planar graphs, as do the proofs of Theorem 1, both of which employ the structural characterisation of $K_{5^{-}}$ minor graphs in terms of planar graphs due to Wagner [16]. The main contribution of this paper is to prove Theorem 1 without using Wagner's characterisation - even without planar embeddings. Given that there is no precise structure theorem for $K_{t}$-minor-free graphs for $t \geq 6$, we consider this a first step towards proving the (Weak) List Hadwiger Conjecture for $t \geq 6$.

\section{Proof of Theorem 1}

Our proof of Theorem 1 is inspired by Thomassen's proof for planar graphs. This remarkable inductive argument allows two adjacent vertices on the outerface to be precoloured (that is, have a list of one colour), the remaining vertices on the outerface have a list of three colours, and the other vertices have a list of five colours. The dependence on the outerface is an obstacle to generalising Thomassen's proof, and motivates the following definition. 
Definition 2. Let $\mathcal{M}$ be a minor-closed class of graphs. Let $B \subseteq V(G)$ for some graph $G \in \mathcal{M}$. Let $G^{B+\alpha}$ be the graph obtained from $G$ by adding a new vertex $\alpha$ adjacent to each vertex in $B$. Then $B$ is an $\mathcal{M}$-boundary of $G$ if $G^{B+\alpha}$ is also in $\mathcal{M}$.

This definition generalises the outerface, since $G^{B+\alpha}$ is planar if and only if all the vertices in $B$ are on the outerface of some planar embedding of $G$.

Lemma 3. Let $\mathcal{M}$ be a minor-closed class of graphs. Let $B$ be an $\mathcal{M}$-boundary of some graph $G \in \mathcal{M}$. Let $v \in B$. Then $C:=(B \backslash\{v\}) \cup N_{G}(v)$ is an $\mathcal{M}$-boundary of $H:=G-v$.

Proof. Observe that $H^{C+\alpha}$ is isomorphic to the graph obtained from $G^{B+\alpha}$ by contracting the edge $v \alpha$. Since $G^{B+\alpha} \in \mathcal{M}$ and $\mathcal{M}$ is minor-closed, $H^{C+\alpha}$ is also in $\mathcal{M}$. That is, $C$ is an $\mathcal{M}$-boundary of $H$.

The following lemma is a corollary of a more general result by Mader [8]; we include the following simple proof for completeness.

Lemma 4. Let $v$ be a vertex in a 2-connected graph $G$. Then $G / v w$ is 2-connected for some edge vw incident to $v$.

Proof. Suppose on the contrary that $G / v w$ is not 2-connected for each edge $v w$ incident to $v$; thus $\{v, w\}$ is a cut set. Choose such an edge $v w$ to minimise the order of a smallest component $H$ of $G-\{v, w\}$. Since $G$ is 2-connected, $v$ has a neighbour $x$ in $H$. Thus $G-\{v, x\}$ contains a component that is a proper subgraph of $H$, which contradicts the choice of $v w$.

Let $x, y, z$ be distinct vertices in a graph $G$. A $K_{3}$-minor rooted at $x, y, z$ consists of three connected subgraphs $X, Y, Z$ of $G$ that are pairwise disjoint and pairwise adjacent, such that $x \in V(X), y \in V(Y)$ and $z \in V(Z)$. See [4, 5, 17] for more on rooted minors. A vertex $v$ of $G$ is good (with respect to $x, y, z$ ) if at least two of $x, y, z$ are in the same component of $G-v$, otherwise $v$ is bad. Note that if $v$ is a vertex in a 2-connected graph $G$, then $G-v$ is connected, and all the vertices in $\{x, y, z\} \backslash\{v\}$ are in the same component of $G-v$; thus at least two of $x, y, z$ are in one component of $G-v$. That is, every vertex is good in a 2-connected graph.

Lemma 5. Let $x, y, z$ be distinct vertices in a graph $G$. Then $G$ has a $K_{3}$-minor rooted at $x, y, z$ if and only if every vertex in $G$ is good.

Proof. ( $\Longrightarrow)$ Let $X, Y, Z$ be the branch sets of a $K_{3}$-minor rooted at $x, y, z$. Let $v \in V(G)$. Without loss of generality, $v \notin X \cup Y$. Since $G[X \cup Y]$ is connected, $x$ and $y$ are in the same component of $G-v$. Thus $v$ is good.

$(\Longleftarrow)$ We proceed by induction. Let $x, y, z$ be distinct vertices in a graph $G$ in which every vertex is good. If $|V(G)|=3$ and $G \not K_{3}$, then without loss of generality, $G$ is a subgraph of the path $(x, y, z)$, implying $y$ is bad. Thus, if $|V(G)|=3$ then $G \cong K_{3}$, and we are done. Now assume that $|V(G)| \geq 4$.

First suppose that $G$ is disconnected. If $x, y$ and $z$ are all in the same component $H$ of $G$, then by induction, $H$ and hence $G$ has a $K_{3}$-minor rooted at $x, y, z$. Otherwise some component contains at most one of $x, y, z$, say $x$. Then $y$ and $z$ are both bad. 
Now assume that $G$ is connected. Suppose that $G$ contains a cut-vertex $v$. Since $v$ is good, at least two of $x, y, z$, say $x$ and $y$, are in the same component of $G-v$. Let $w$ be a neighbour of $v$ in a component of $G-v$ not containing $x$ and $y$. Let $G^{\prime}$ be the graph obtained from $G$ by contracting $v w$ into a vertex $v^{\prime}$. We may consider $x, y, z$ to be vertices of $G^{\prime}$. (It is possible that $w=z$ and $v^{\prime}=z$.) In $G^{\prime}$, the vertex $v^{\prime}$ is good since $x$ and $y$ remain in the same component of $G^{\prime}-v^{\prime}$. If some other vertex in $G^{\prime}$ is bad, then it would be bad in $G$. Thus every vertex in $G^{\prime}$ is good. By induction, $G^{\prime}$ and hence $G$ contains a $K_{3}$-minor rooted at $x, y, z$.

Now assume that $G$ is 2-connected. Choose $v \in V(G) \backslash\{x, y, z\}$. By Lemma 4, $G / v w$ is 2-connected for some edge $v w$ incident to $v$. Thus every vertex is good in $G / v w$. Since $x, y, z$ are distinct vertices in $G / v w$, by induction, $G / v w$ and hence $G$ has a $K_{3}$-minor rooted at $x, y, z$.

A graph $G$ is said to contain every rooted $K_{3}$-minor if $G$ contains a $K_{3}$-minor rooted at $x, y, z$ for all distinct $x, y, z \in V(G)$.

Proposition 6. A graph $G$ contains every rooted $K_{3}$-minor if and only if $G$ is 2 connected.

Proof. Since every vertex is good in a 2-connected graph, by Lemma 5, a 2-connected graph contains every rooted $K_{3}$-minor. For the converse, let $G$ be a graph that contains every rooted $K_{3}$-minor. If $G$ is disconnected, then there is no $K_{3}$-minor rooted at $x, y, z$, whenever $x$ and $y$ are in distinct components. Hence $G$ is connected. If $G$ has a cutvertex $x$, then $G$ contains no $K_{3}$-minor rooted at $x, y, z$, whenever $y$ and $z$ are in distinct components of $G-x$. Hence $G$ is 2-connected.

Let $G_{1}$ and $G_{2}$ be subgraphs of a graph $G$, such that $G=G_{1} \cup G_{2}$ and $V\left(G_{1}\right) \backslash V\left(G_{2}\right) \neq$ $\emptyset$ and $V\left(G_{2}\right) \backslash V\left(G_{1}\right) \neq \emptyset$. In particular, there is no edge between $V\left(G_{1}\right) \backslash V\left(G_{2}\right)$ and $V\left(G_{2}\right) \backslash V\left(G_{1}\right)$. Then $\left\{G_{1}, G_{2}\right\}$ is a separation of order $\left|V\left(G_{1}\right) \cap V\left(G_{2}\right)\right|$.

Theorem 1 is a consequence of the following lemma (with $A=B=\emptyset$ ).

Lemma 7. Let $\mathcal{M}$ be the class of $K_{5}$-minor-free graphs. Let $G \in \mathcal{M}$. Let $A \subseteq B \subseteq V(G)$, such that $A$ is a clique, and $B$ is an $\mathcal{M}$-boundary of $G$. Let $L$ be a list-assignment of $G$ such that:

- $|L(x)|=1$ for each vertex $x \in A$,

- $L(x) \neq L(y)$ for distinct $x, y \in A$,

- $|L(x)| \geq 3$ for each vertex $x \in B \backslash A$,

- $|L(x)| \geq 5$ for each vertex $x \in V(G) \backslash B$.

Then $G$ is L-colourable.

Proof. Let $(G, A, B, L)$ be a counterexample with $|V(G)|$ minimum, and then with $|A|$ maximum. Clearly $|V(G)| \geq 4$.

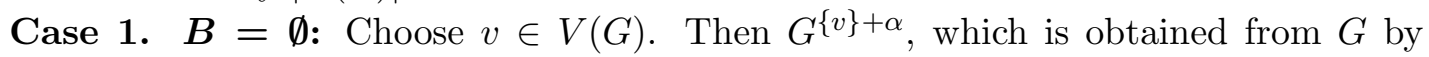
adding a new vertex $\alpha$ adjacent to $v$, is $K_{5}$-minor-free. Let $L^{\prime}(v):=\{c\}$ for some colour $c \in L(v)$. Let $L^{\prime}(x):=L(x)$ for every other vertex $x$. By the choice of $(G, A, B, L)$, the 
instance $\left(G,\{v\},\{v\}, L^{\prime}\right)$ is not a counterexample, and $G$ is $L$-colourable. Now assume that $B \neq \emptyset$.

Case 2. $\boldsymbol{A}=\emptyset$ : Choose $v \in B$. Let $L^{\prime}(v):=\{c\}$ for some colour $c \in L(v)$. Let $L^{\prime}(x):=L(x)$ for every other vertex $x$. Again $\left(G,\{v\}, B, L^{\prime}\right)$ is not a counterexample, and $G$ is $L$-colourable. Now assume that $A \neq \emptyset$.

Case 3. $G$ is not connected: Then $G$ contains a separation $\left\{G_{1}, G_{2}\right\}$ with $V\left(G_{1} \cap\right.$ $\left.G_{2}\right)=\emptyset$. Since $A$ is a clique, without loss of generality, $A \subseteq V\left(G_{1}\right)$. Let $B_{i}:=B \cap V\left(G_{i}\right)$. Then $B_{i}$ is an $\mathcal{M}$-boundary of $G_{i}$ (since $G_{i}{ }^{B_{i}+\alpha} \subseteq G^{B+\alpha}$ ). Define $L_{i}(x):=L(x)$ for each vertex $x$ in $G_{i}$. Hence $\left(G_{1}, A, B_{1}, L_{1}\right)$ is not a counterexample, and $G_{1}$ is $L_{1}$-colourable. Also $\left(G_{2}, \emptyset, B_{2}, L_{2}\right)$ is not a counterexample, and $G_{2}$ is $L_{2}$-colourable. Hence $G$ is $L$ colourable. Now assume that $G$ is connected.

Case 4. $\boldsymbol{G}$ contains a cut-vertex $\boldsymbol{v} \in \boldsymbol{B}$ : Then $G$ contains a separation $\left\{G_{1}, G_{2}\right\}$ with $V\left(G_{1} \cap G_{2}\right)=\{v\}$. Since $A$ is a clique, without loss of generality, $A \subseteq V\left(G_{1}\right)$. Let $B_{i}:=B \cap V\left(G_{i}\right)$. Then $B_{i}$ is an $\mathcal{M}$-boundary of $G_{i}$. Define $L_{1}(x):=L(x)$ for each vertex $x$ in $G_{1}$. Thus $\left(G_{1}, A, B_{1}, L_{1}\right)$ is not a counterexample, and $G_{1}$ is $L_{1}$-colourable. Let $L_{2}(v):=\{c\}$ where $v$ is coloured $c$ in $G_{1}$. Let $L_{2}(x):=L(x)$ for every other vertex $x$ in $G_{2}$. Then $\left(G_{2},\{v\}, B_{2}, L_{2}\right)$ is not a counterexample (since $v \in B_{2}$ ), and $G_{2}$ is $L_{2^{-}}$ colourable. Hence $G$ is $L$-colourable (since $v$ receives the same colour in $G_{1}$ and in $G_{2}$, and each edge of $G$ is in $G_{1}$ or $G_{2}$ ). Now assume that $G-v$ is connected for every vertex $v \in B$.

Case 5. $G$ contains a cut-vertex $v$ separating two vertices in $B$ : Then $G$ contains a separation $\left\{G_{1}, G_{2}\right\}$ with $V\left(G_{1} \cap G_{2}\right)=\{v\}$, such that $B \cap V\left(G_{1}-v\right) \neq \emptyset$ and $B \cap V\left(G_{2}-v\right) \neq \emptyset$. Since $A$ is a clique, without loss of generality, $A \subseteq V\left(G_{1}\right)$. Let $B_{1}:=B \cap V\left(G_{1}\right)$. Then $B_{1}$ is an $\mathcal{M}$-boundary of $G_{1}$. Define $L_{1}(x):=L(x)$ for each vertex $x$ in $G_{1}$. Thus $\left(G_{1}, A, B_{1}, L_{1}\right)$ is not a counterexample, and $G_{1}$ is $L_{1}$-colourable.

Since $G$ is connected, $G_{1}$ is connected. Let $B_{2}:=\left(B \cap V\left(G_{2}\right)\right) \cup\{v\}$. Then $B_{2}$ is an $\mathcal{M}$-boundary of $G_{2}$, since $G_{2}{ }^{B_{2}+\alpha}$ is a minor of $G^{B+\alpha}$ obtained by contracting $G_{1}$ into $v$ (since $\alpha$ has a neighbour in $G_{1}-v$ ). Let $L_{2}(v):=\{c\}$ where $v$ is coloured $c$ in $G_{1}$. Let $L_{2}(x):=L(x)$ for every other vertex $x$ in $G_{2}$. Then $\left(G_{2},\{v\}, B_{2}, L_{2}\right)$ is not a counterexample (since $v \in B_{2}$ ), and $G_{2}$ is $L_{2}$-colourable. Hence $G$ is $L$-colourable. Now assume that $G$ contains no cut-vertex separating two vertices in $B$.

Case 6. $G$ contains a cut-set $\{v, w\}$ separating two vertices in $B$, where $\boldsymbol{v} \in \boldsymbol{B}$ : Thus $G$ has a separation $\left\{G_{1}, G_{2}\right\}$ with $V\left(G_{1}\right) \cap V\left(G_{2}\right)=\{v, w\}$ and $B \cap V\left(G_{1}-\right.$ $\{v, w\}) \neq \emptyset$ and $B \cap V\left(G_{2}-\{v, w\}\right) \neq \emptyset$.

Suppose that $v w \notin E(G)$. We claim that adding the edge $v w$ creates no $K_{5}$-minor in $G^{B+\alpha}$. Let $G^{\prime}$ be the graph obtained from $G^{B+\alpha}$ by adding the edge $v w$. Let $H$ be a 4-connected minor in $G^{\prime}$. Since $\{\alpha, v, w\}$ is a separator in $G^{\prime}$, there are no two branch sets of $H$, with one contained in $V\left(G_{1}\right) \backslash V\left(G_{2}\right)$, and the other contained in $V\left(G_{2}\right) \backslash V\left(G_{1}\right)$. Thus, without loss of generality, every branch set of $H$ intersects $G_{1}$. By Cases 4 and 5 , neither $v$ nor $w$ are cut-vertices in $G$. Thus there is $v w$-path $P$ in $G_{2}$. Hence the edge $v w$ in our $H$-minor can be replaced by $P$ to obtain an $H$-minor in $G^{B+\alpha}$ (without $v w$ ). Since $K_{5}$ is 4 -connected and $G^{B+\alpha}$ is $K_{5}$-minor-free, $G^{\prime}$ is also $K_{5}$-minor-free. That is, adding 
$v w$ does not create a $K_{5}$-minor in $G^{B+\alpha}$ (and also not in $G$ ). Since $A$ is a clique, adding $v w$ does not break any of the assumptions in the lemma. Now assume that $v w \in E(G)$.

Since $A$ is a clique, without loss of generality, $A \subseteq V\left(G_{1}\right)$. Let $B_{1}:=B \cap V\left(G_{1}\right)$. Then $B_{1}$ is an $\mathcal{M}$-boundary of $G_{1}$. Define $L_{1}(x):=L(x)$ for each vertex $x$ in $G_{1}$. Thus $\left(G_{1}, A, B_{1}, L_{1}\right)$ is not a counterexample, and $G_{1}$ is $L_{1}$-colourable.

Since $G$ is connected and $v$ is not a cut-vertex, $G_{1}-v$ is connected. Let $B_{2}:=$ $\left(B \cap V\left(G_{2}\right)\right) \cup\{w\}$. Then $B_{2}$ is an $\mathcal{M}$-boundary of $G_{2}$, since $G_{2}{ }^{B_{2}+\alpha}$ is a minor of $G^{B+\alpha}$ obtained by contracting $G_{1}-v$ into $w$ (since $\alpha$ has a neighbour in $G_{1}-v$ ). Let $L_{2}(v):=\{c\}$ where $v$ is coloured $c$ in $G_{1}$. Let $L_{2}(w):=\{d\}$ where $w$ is coloured $d$ in $G_{1}$. Let $L_{2}(x):=L(x)$ for every other vertex $x$ in $G_{2}$. Then $\left(G_{2},\{v, w\}, B_{2}, L_{2}\right)$ is not a counterexample (since $\{v, w\} \subseteq B_{2}$ ), and $G_{2}$ is $L_{2}$-colourable. Hence $G$ is $L$-colourable. Now assume that $G$ contains no such cut-set $\{v, w\}$.

Case 7. Some vertex $\boldsymbol{v} \in \boldsymbol{B}$ has degree at least 3 in $\boldsymbol{G}[\boldsymbol{B}]:$ Let $x, y, z$ be three neighbours of $v$ in $B$. If $G-v$ contains a $K_{3}$-minor rooted at $x, y, z$, then adding $v$ and $\alpha$ gives a $K_{5}$-minor in $G^{B+\alpha}$, which contradicts the assumption that $B$ is an $\mathcal{M}$-boundary of $G$. Thus $G-v$ contains no $K_{3}$-minor rooted at $x, y, z$. By Lemma 5 , for some vertex $w$ in $G-v$, all the vertices in $\{x, y, z\} \backslash\{w\}$ are in distinct components of $G-\{v, w\}$. Thus $\{v, w\}$ is a cut-set satisfying Case 6 or 7 . Now assume that $G[B]$ has maximum degree at most 2 .

Case 8. $\boldsymbol{G}[\boldsymbol{A}]$ is a component of $\boldsymbol{G}[\boldsymbol{B}]$ : Choose $v \in A$. Let $G^{\prime}:=G-v$ and $A^{\prime}:=A \backslash\{v\}$ and $B^{\prime}:=(B \backslash\{v\}) \cup N_{G}(v)$. By Lemma 3, $B^{\prime}$ is an $\mathcal{M}$-boundary of $G^{\prime}$. Let $L^{\prime}(u):=L(u) \backslash L(v)$ for each vertex $u \in N_{G}(v) \backslash B$. Since $|L(v)|=1$ and $v$ has no neighbour in $B \backslash A$, we have $\left|L^{\prime}(x)\right| \geq 3$ for each $x \in B^{\prime}$. Let $L^{\prime}(x):=L(x)$ for every other vertex $x$. Then $\left(G^{\prime}, A^{\prime}, B^{\prime}, L^{\prime}\right)$ is not a counterexample, and $G^{\prime}$ is $L^{\prime}$-colourable. Assign $v$ the colour in $L(v)$. This colour is not in $L^{\prime}(u)$ for each $u \in N_{G}(v)$. Thus $G$ is $L$-colourable.

Case 9. $\boldsymbol{G}[\boldsymbol{A}]$ is not a component of $\boldsymbol{G}[\boldsymbol{B}]$ : Choose $v \in B \backslash A$ adjacent to some vertex $p \in A$. Since $G[B]$ has maximum degree at most $2, v$ has at most one other neighbour in $B$; let $w$ be this neighbour (if it exists). Let $G^{\prime}:=G-v$ and $B^{\prime}:=(B \backslash\{v\}) \cup N_{G}(v)$. By Lemma 3, $B^{\prime}$ is an $\mathcal{M}$-boundary of $G^{\prime}$. Let $c, d$ be distinct colours in $L(v) \backslash L(p)$. Let $L^{\prime}(u):=L(u) \backslash\{c, d\}$ for each vertex $u \in N_{G}(v) \backslash B$; thus $\left|L^{\prime}(u)\right| \geq 5-2=3$. Let $L^{\prime}(x):=L(x)$ for every other vertex $x$. Then $\left(G^{\prime}, A, B^{\prime}, L^{\prime}\right)$ is not a counterexample, and $G^{\prime}$ is $L^{\prime}$-colourable. Assign $v$ colour $c$ or $d$ different from the colour assigned to $w$ (if $w$ exists). Hence $G$ is $L$-colourable.

\section{REFERENCES}

[1] Reinhard Diestel. Graph theory, vol. 173 of Graduate Texts in Mathematics. Springer, 3rd edn., 2005. http://diestel-graph-theory.com/index.html.

[2] Hugo Hadwiger. Über eine Klassifikation der Streckenkomplexe. Vierteljschr. Naturforsch. Ges. Zürich, 88:133-142, 1943. 
[3] Wenjie He, Wenjing Miao, and Yufa Shen. Another proof of the 5choosability of $K_{5}$-minor-free graphs. Discrete Math., 308(17):4024-4026, 2008. http://dx.doi.org/10.1016/j.disc.2007.07.089.

[4] Leif K. JøRGensen AND Ken-ICHi KaWARAbayashi. Extremal results for rooted minor problems. J. Graph Theory, 55(3):191-207, 2007. http://dx.doi.org/10.1002/jgt.20232.

[5] Ken-IChi KaWARABayashi. Rooted minor problems in highly connected graphs. Discrete Math., 287(1-3):121-123, 2004. http://dx.doi.org/10.1016/j.disc.2004.07.007.

[6] Ken-IChi Kawarabayashi and Bojan Mohar. A relaxed Hadwiger's conjecture for list colorings. J. Combin. Theory Ser. B, 97(4):647-651, 2007. http://dx.doi.org/10.1016/j.jctb.2006.11.002.

[7] Alexandr V. Kostochka. The minimum Hadwiger number for graphs with a given mean degree of vertices. Metody Diskret. Analiz., 38:37-58, 1982.

[8] Wolfgang Mader. Generalizations of critical connectivity of graphs. Discrete Math., 72(1-3):267-283, 1988. http://dx.doi.org/10.1016/0012-365X (88)90216-6.

[9] Neil Robertson, Daniel P. Sanders, Paul D. Seymour, and Robin Thomas. The four-colour theorem. J. Combin. Theory Ser. B, 70(1):2-44, 1997. http://dx.doi.org/10.1006/jctb.1997.1750.

[10] Neil Robertson, Paul D. Seymour, and Robin Thomas. Hadwiger's conjecture for $K_{6}$-free graphs. Combinatorica, 13(3):279-361, 1993. http://dx.doi.org/10.1007/BF01202354.

[11] Riste Škrekovski. Choosability of $K_{5}$-minor-free graphs. Discrete Math., 190(13):223-226, 1998. http://dx.doi.org/10.1016/S0012-365X (98)00158-7.

[12] Andrew Thomason. An extremal function for contractions of graphs. Math. Proc. Cambridge Philos. Soc., 95(2):261-265, 1984. http://dx.doi.org/10.1017/S0305004100061521.

[13] Carsten Thomassen. Every planar graph is 5-choosable. J. Combin. Theory Ser. B, 62(1):180-181, 1994. http://dx.doi.org/10.1006/jctb.1994.1062.

[14] Bjarne Toft. A survey of Hadwiger's conjecture. Congr. Numer., 115:249-283, 1996.

[15] Margit Voigt. List colourings of planar graphs. Discrete Math., 120(1-3):215-219, 1993. http://dx.doi.org/10.1016/0012-365X(93)90579-I.

[16] Klaus Wagner. Über eine Eigenschaft der ebene Komplexe. Math. Ann., 114:570590, 1937. http://dx.doi.org/10.1007/BF01594196.

[17] Paul Wollan. Extremal functions for rooted minors. J. Graph Theory, 58(2):159178, 2008. http://dx.doi.org/10.1002/jgt.20301.

[18] David R. Wood. Contractibility and the Hadwiger conjecture. European J. Combin., 31(8):2102-2109, 2010. http://dx.doi.org/10.1016/j.ejc.2010.05.015

[19] Douglas R. Woodall. List colourings of graphs. In Surveys in combinatorics, vol. 288 of London Math. Soc. Lecture Note Ser., pp. 269-301. Cambridge Univ. 
Press, 2001.

Department of Mathematics and Statistics

The University of Melbourne

Melbourne, Australia

E-mail address: woodd@unimelb.edu.au

Department of Mathematics

KTH-Royal Institute of TeChnology

STOCKHolm, SwEDEN

E-mail address: linusson@math.kth.se 